\title{
Downregulation of Id1 by small interfering RNA in gastric cancer inhibits cell growth via the Akt pathway
}

\author{
GUANG YANG $^{1,3}$, YAN ZHANG ${ }^{4}$, JIANJUN XIONG $^{1}$, JING WU $^{1}$, \\ CHANGFU YANG ${ }^{1,3}$, HONGBING HUANG $^{2}$ and ZHENYU ZHU ${ }^{1,3}$
}

\author{
${ }^{1}$ Department of Biochemistry and Molecular Biology, Zhongshan Medical College; ${ }^{2}$ Department of Pharmacy, \\ State Key Laboratory of Oncology in South China, Cancer Center; ${ }^{3}$ DaAn Gene Co., Ltd., Sun Yat-Sen University, Guangzhou; \\ ${ }^{4}$ The Center of Prenatal Diagnosis, Maternal and Child Health Hospital of Guangdong Province, Guangdong, P.R. China
}

Received September 13, 2011; Accepted December 27, 2011

DOI: $10.3892 / \mathrm{mmr} .2012 .749$

\begin{abstract}
Inhibitor of differentiation or DNA binding (Id1) is a member of the helix-loop-helix transcription factor family that is overexpressed in various types of cancer, including gastric carcinoma. Previous studies showed that Id1 is a prognostic marker in patients with gastric cancer. However, the role of Id1 in the proliferation of human gastric cancer cells has yet to be clarified. In the present study, we downregulated the Id1 gene in SGC-7901 gastric cancer cells by RNA interference, and we also constructed a recombinant plasmid-expressing Id1 to investigate its effects on the proliferation of SGC-7901 cells. Results showed that the downregulation of Id1 inhibited proliferation of SGC-7901 cells, while the upregulation of Id1 had no effect on SGC-7901 cell proliferation. The potential mechanism was also investigated. The changes of certain proteins associated with cell proliferation, apoptosis and the cell cycle were detected by western blotting. Furthermore, we demonstrated a positive correlation between Id1 and phospho-Akt expression in SGC-7901 cells.
\end{abstract}

\section{Introduction}

Gastric carcinoma is a common disease with high incidence rates in several Asian countries, particularly in Japan and China. Lower incidence has been observed in certain Western European countries and the United States $(1,2)$. Although the

Correspondence to: Dr Hongbing Huang, Department of Pharmacy, State Key Laboratory of Oncology in South China, Cancer Center, Sun Yat-Sen University, 74, Zhongshan Road, Guangzhou, Guangdong 510080, P.R. China

E-mail: hhongb@mail.sysu.edu.cn

Dr Zhenyu Zhu, Department of Biochemistry and Molecular Biology, Zhongshan Medical College, Sun Yat-Sen University, 74, Zhongshan Road, Guangzhou, Guangdong 510080, P.R. China

E-mail: yangpecker2@yahoo.com.cn

Key words: inhibitor of differentiation or DNA binding, gastric cancer, growth, RNA interference, Akt pathway incidence of gastric carcinoma has decreased in recent years, it remains the second cause of cancer-related death worldwide (3). Due to the majority of the cases being detected at advanced stages, the 5-year survival rate in these cases is low (4). Therefore, it is imperative to find new targets to improve therapeutic or preventive strategies.

Inhibitor of DNA binding 1 (Id1) belongs to the inhibitor of DNA binding/differentiation (Id) family, which lacks a DNA-binding domain (5), so it acts as a negative regulator of HLH transcription factors to inhibit gene expression $(6,7)$. Id1 was previously reported to regulate various cell processes, including proliferation, apoptosis, cell cycle, differentiation and angiogenesis (8-11). The upregulation of Id1 may inhibit the ability to differentiate in several cell models. Certain reports have suggested that cell cycle-associated proteins, such as p16, p21, p27 and cyclin D1, are transcriptionally inhibited by Id1; the upregulation of Id1 may stimulate G1-S cell cycle transition (12-14). The role of Id1 in cell proliferation or apoptosis showed different effects in different cell types: the upregulation of Id1 induces apoptosis in dense mammary epithelial cells and cardiac myocytes, but promotes proliferation and tumor growth in lung cancer cells (14-16). Id1 is regarded as a valuable marker for both the diagnosis and prognosis of gastric carcinoma $(17,18)$. Although several reports have suggested that Id1 is involved in the growth and migration of gastric cancer cells (19), the role of Id1 in the proliferation and migration abilities of gastric cancer cells remains to be determined.

In this study, we mainly investigated the role of Id1 in the proliferation of SGC-7901 cells by knockdown and overexpression techniques, and a possible mechanism was also found. Our findings indicated that Id1 is involved in the growth and migration abilities of gastric cancer cells.

\section{Materials and methods}

Cell culture. The SGC-7901 gastric cancer cell line was a gift from Dr Yang Zhang (Department of Biochemistry and Molecular Biology, Zhongshan Medical College, Sun Yat-Sen University, China) (20). The cell line was cultured in high-glucose DMEM (Gibco, BRL, Guangzhou, China) supplemented with $10 \%$ fetal bovine serum at $37^{\circ} \mathrm{C}$ with $5 \% \mathrm{CO}_{2}$. 
A

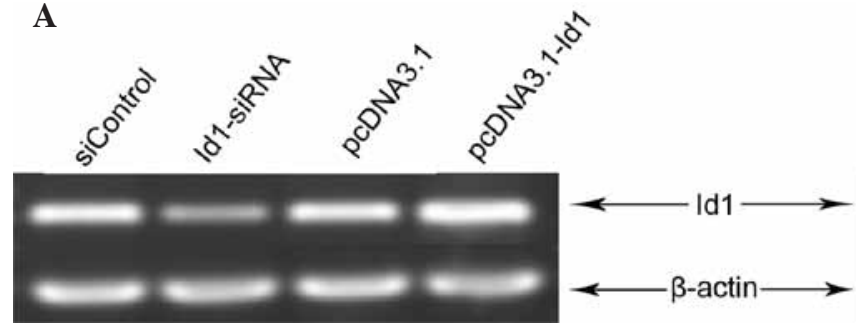

B

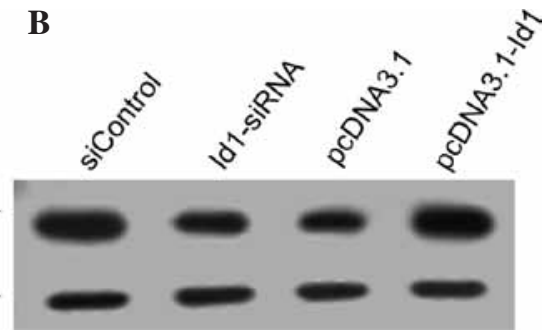

Figure 1. Levels of Id1 mRNA and protein in SGC-7901 cells transfected with Id-siRNA or pcDNA3.1-Id1 for 72 h. (A) RT-PCR assay indicated that Id1siRNA potently decreased the quantity of Id1 mRNA, while pcDNA3.1-Id1 increased the mRNA in the SGC7901 cells. (B) Western blotting indicated that the protein levels of Id1 in SGC-7901 cells were successfully regulated by Id1-siRNA or pcDNA3.1-Id1. $\beta$-actin was used as an internal loading control.

Id1 small interfering RNA (siRNA). Id1-specific siRNA used for Id1 knockdown and the control siRNA were synthesized by GenePharma (Shanghai GenePharma Co., Ltd.). The sequences of siRNA targeting the Id1 coding region were as follows: sense, 5'-CUCGGAAUCCGAAGUUGGADTDT-3' and antisense, 5'-UCCAACUUCGGAUUCCGAGDTDT-3' (21). The siRNAs were then transfected into the PC3 cells by Lipofectine 2000 (Invitrogen, USA), according to the manufacturer's instructions.

Construction of the Idl expressing vector. The full-length Id1 cDNA was amplified from total cDNA of SGC-7901 cells by PCR, and was then subcloned between the KpnI and EcoRI sites of pcDNA3.1(+) vector. Purified plasmids were sequence-verified by Invitrogen (Shanghai, China). The plasmid was transfected into SGC-7901 cells by Lipofectine 2000. The primers used for PCR were as follows: forward, 5'-GATGGTACCATCATGAAAGTCGCCAGTG-3' and reverse, 5'-GATGAATTCTCAGCGACACAAGATGCGA-3'.

MTT assay. SGC-7901 cells were seeded in 96-well plates at a concentration of 5,000 cells/well in a volume of $150 \mu \mathrm{l}$ of cell culture medium. After $24 \mathrm{~h}$, transfection was performed. The plates were incubated at $37^{\circ} \mathrm{C}$ with $5 \% \mathrm{CO}_{2}$ for 48 and $72 \mathrm{~h}$. MTT solution $(20 \mu \mathrm{l})(5 \mathrm{~g} / \mathrm{l}$, dissolved in PBS) was added to each well and the plates were incubated at $37^{\circ} \mathrm{C}$ for another $4 \mathrm{~h}$. Subsequently, the supernatant was discarded and $150 \mu \mathrm{l}$ dimethylsulfoxide was added to dissolve the insoluble MTT formazan. The absorbance values at $570 \mathrm{~nm}$ were detected by a multi-well plate reader (Tecan).

Flow cytometry assay. SGC-7901 cells were transfected with the above-mentioned siRNA or plasmid for $72 \mathrm{~h}$ and were then presented for flow cytometry assay. For cell cycle analysis, DNA labeling was performed using the Cycletest Plus DNA Reagent kit (BD Biosciences Pharmingen, USA), and the samples were analyzed using a flow cytometer (Beckman Counter, USA). For the detection of apoptotic cells, labeling tests involving both propidium iodide (PI) and annexin-V were performed using an Annexin-V staining kit (Invitrogen, USA), according to the manufacturer's instructions. Briefly, at least $1 \times 10^{6}$ cells were harvested by trypsinization, incubated with FITC-labeled annexin-V and PI stock solutions for $10 \mathrm{~min}$ at room temperature and analyzed using a flow cytometer (Beckman Counter).

Western blot analysis. SGC-7901 cells were transfected for $72 \mathrm{~h}$, harvested and lysed for total protein extraction. Protein concentration was determined using the Bio-Rad protein assay kit (Bio-Rad, China). Equal amounts of protein were separated by $15 \%$ SDS-PAGE and transferred onto PVDF membranes. The membranes were rinsed with TBST and incubated in blocking buffer (5\% dried milk in PBS) for $1 \mathrm{~h}$ at $37^{\circ} \mathrm{C}$, followed by incubation with primary antibodies at $4^{\circ} \mathrm{C}$ overnight. The antibody against cyclin D1 used in western blotting was purchased from Beyotime (China), antibody against $\beta$-actin was purchased from PTG (USA), and other antibodies were purchased from Santa Cruz (USA). After washing with TBST three times, the membranes were incubated with their corresponding secondary antibodies for $1 \mathrm{~h}$. The blots were visualized by an enhanced chemiluminescence detection system (Amersham). The expression of $\beta$-actin was used as a normalization control for protein loading.

Statistical analysis. Data were expressed as the means \pm SD. Statistical analyses were performed using Student's t-test. $\mathrm{P}<0.05$ indicated statistical significance.

\section{Results}

Effects of Id1 siRNA or pcDNA3.1-Id1 on the levels of Id1 in $S G C-7901$ cells. The effect of siRNA or pcDNA3.1-Id1 on the levels of Id1 was evaluated using both reverse transcriptasePCR and western blotting. As shown in Fig. 1, significantly decreased Id 1 mRNA and protein levels were detected in siRNA transfected cells compared to the control group (siControl), indicating that Id1 siRNA successfully downregulated the Id1 gene in SGC-7901 cells. Furthermore, pcDNA3.1-Id1 upregulated the levels of Id1 in SGC-7901 cells.

Regulation of SGC-7901 cell proliferation by Idl expression. To evaluate the effect of Id1 on the proliferation of SGC-7901 cells, MTT assay was performed. As shown in Fig. 2, Id1 was involved in the proliferation of SGC-7901 cells; the inhibitory effect was evident after 72-h transfection with Id1-siRNA. However, the upregulation of Id1 in SGC-7901 cells could not promote cell proliferation.

Regulation of Id1 did not affect the apoptosis of SGC-7901 cells. To examine the effect of altered Id1 expression on cell apoptosis, transfected cells were analyzed by Annexin V-FITC/ PI double staining. As shown in Fig. 3A, compared to the control group, no significant change was found in the annexin V-positive or annexin V and PI-double positive cell fractions in the Id1 siRNA-transfected group. Moreover, Id1 overexpression 
A

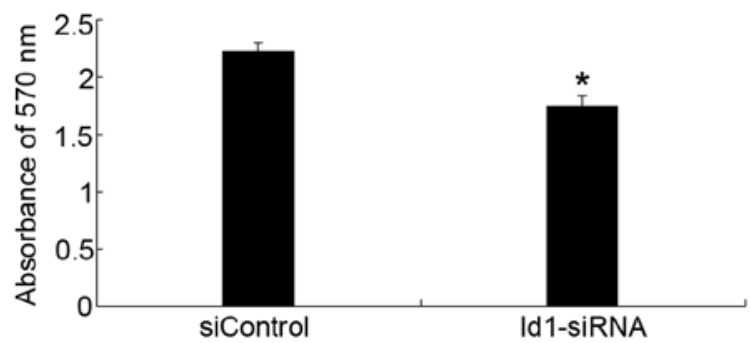

B

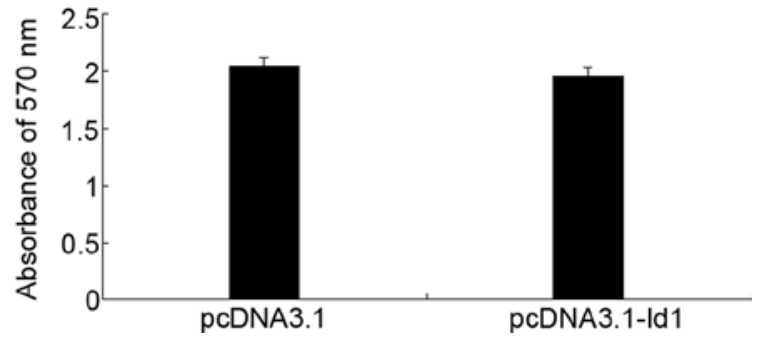

Figure 2. MTT assay was performed when the cells were transfected for $72 \mathrm{~h}$. (A) Id1-siRNA showed inhibitory effects on the proliferation of SGC-7901 cells. (B) Upregulation of Id1 in SGC-7901 cells showed no affect on cell proliferation. Values are the means \pm SD of 3 wells. *P<0.05 compared to the control group cells.

A

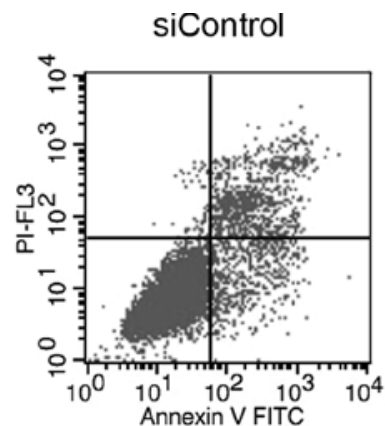

B

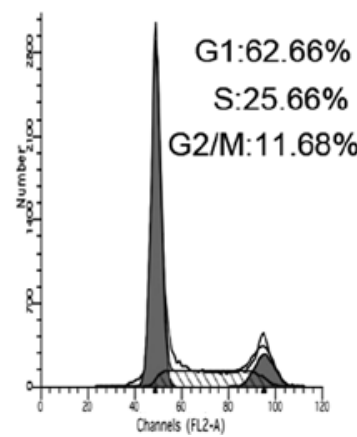

Id1-siRNA
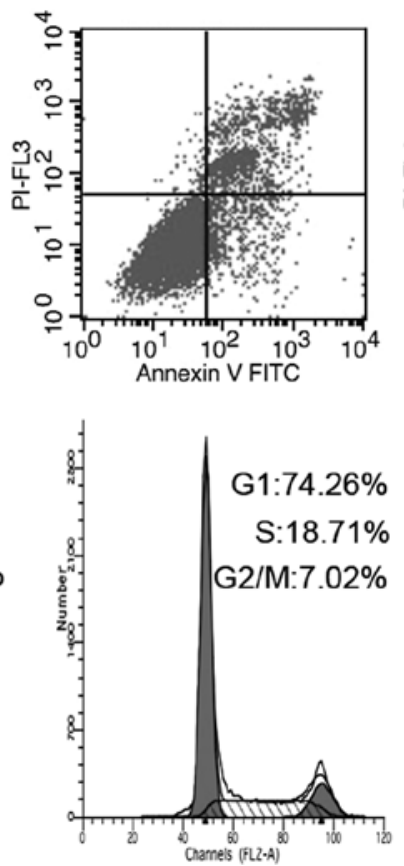

pcDNA3.1
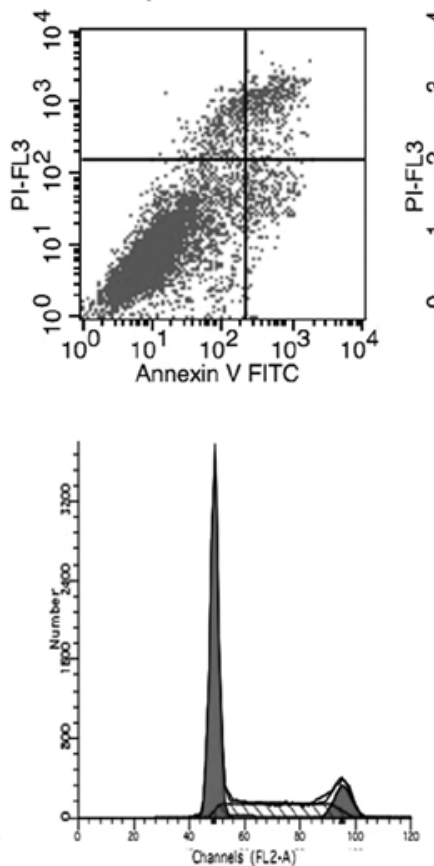

pcDNA3.1-Id 1
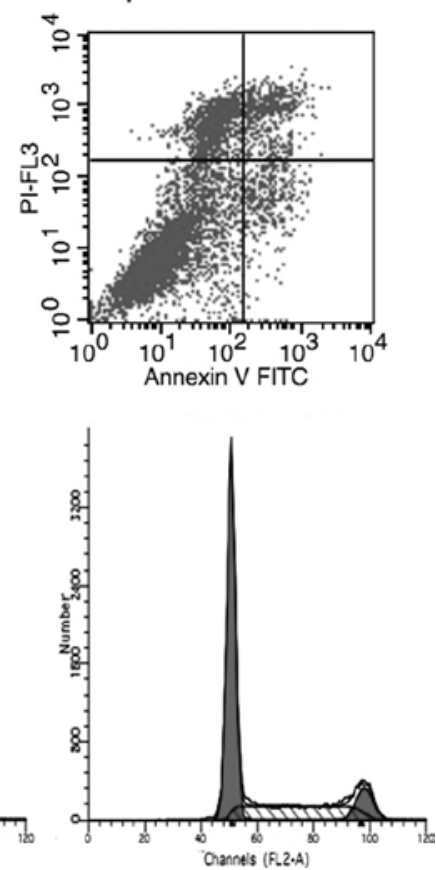

Figure 3. Flow cytometry assays were performed to detect the changes of apoptosis and the cell cycle of SGC-7901 cells transfected with the siRNA or plasmids decribed in Materials and Methods. (A) Regulating the levels of Id1 in SGC-7901 cells did not affect cell apoptosis. (B) Downregulation of Id1 in SGC-7901 cells decreased the proportion of the G2/M phase in the cell cycle, while the upregulation of Id1 did not exert this effect on SGC-7901 cells.

in SGC-7901 cells transfected with pcDNA3.1-Id1 showed no impact on cell apoptosis; it suggested that Id1 is not involved in the apoptosis of SGC-7901 cells. Bax and Bad are important in the apoptotic pathway. In this study, although the changes of Id1 levels did not affect the apoptosis of SGC-7901 cells, we detected the protein levels of Bax and Bad in SGC-7901 cells. As shown in Fig. 4A, the expression of Bax and Bad increased when cells were transfected with Id1 siRNA, as expected, whereas their expression decreased when cells were transfected with pcDNA3.1-Id1.

Effects of Idl expression on cell cycle distribution. Cell cycle distribution was analyzed by flow cytometry after the cells were transfected for $72 \mathrm{~h}$. As shown in Fig. 3B, the proportion of the G2/M phase was decreased in Id1 siRNA-transfected cells compared to the control group. The cell cycle did not change when Id1 was overexpressed in SGC-7901 cells. We further analyzed the changes of certain cell cycle regulators. Cyclin D1 was decreased in the Id1 knockdown group, while p16 and p21 were increased; cyclin D1 was elevated in the
Id1 overexpression group, while p16 and p21 were decreased (Fig. 4B).

Akt pathway is involved in the growth inhibition of SGC-7901 cells following transfection with IdI siRNA. The correlation between Id1 and p-Akt expression in SGC-7901 cells was examined by western blotting. As shown in Fig. 5, Akt phosphorylation at Thr308 and Ser473 was reduced in Id1 siRNA-treated SGC-7901 cells, but enhanced in pcDNA3.1Id1-treated SGC-7901 cells. These results suggest that the expression of p-Akt is associated with Id1 expression in SGC-7901 cells.

\section{Discussion}

Id proteins are involved in cell differentiation, proliferation, migration and angiogenesis. Recent studies have investigated the role of Id1 in cancer development in several tumor models $(22,23)$. Data on its role in gastric carcinoma remain scarce and are derived mainly from tissue resources $(17,18)$. A previous 
A

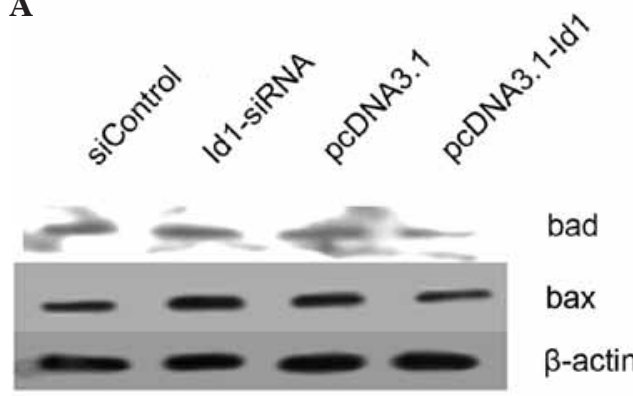

B

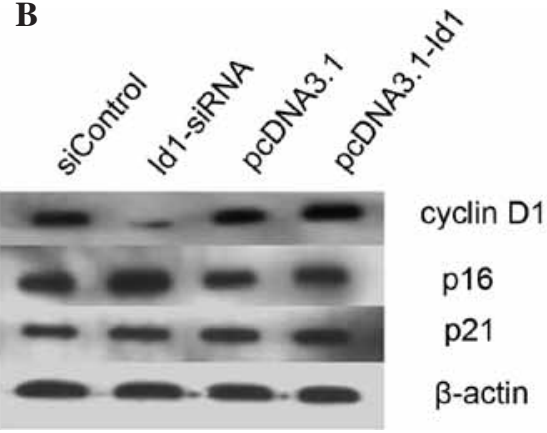

Figure 4. Western blotting was performed to detect the quantity changes of certain apoptosis-related genes or cell cycle-related genes when SGC-7901 cells were transfected with Id1-siRNA or pcDNA3.1-Id1. (A) The levels of both Bax and Bad were elevated in Id1 downregulated SGC-7901 cells, but decreased in the Id1 upregulated groups. (B) p16 and p21 were increased in the Id1 downregulated SGC-7901 cells, but decreased in the Id1 up-regulated groups. Cyclin D1 was decreased in Id1 knockdown SGC-7901 cells, but increased in the Id1 overexpressing group. $\beta$-actin was used as an internal loading control.

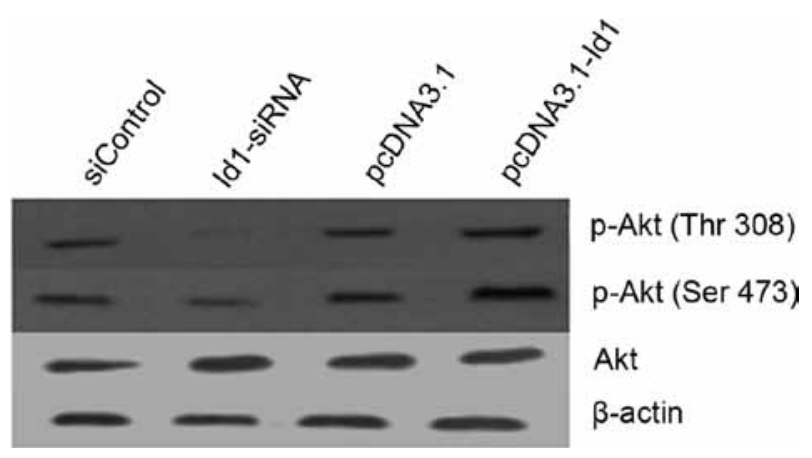

Figure 5. The Akt pathway was involved in Id1-associated SGC-7901 cell proliferation. Western blotting results showed that the quantity of p-Akt (Thr308, Ser473) was reduced in Id1 knockdown SGC-7901 cells, while overexpression of Id1 elevated the levels of p-Akt (Thr308, Ser473). $\beta$-actin was used as an internal loading control.

report has shown that Id1, 3 double-knockdown impaired the ability of gastric cancer cells to form peritoneal metastasis. Findings of that study also suggested that proliferation and motility may be inhibited in Id1, 3 double-knockdown gastric cancer cells (19). However, the single role of Id1 in gastric cancer cells was not investigated.

We investigated the role of Id1 in the proliferation of gastric carcinoma by changing the Id1 levels in SGC-7901 cells. Id1-siRNA and the vector expressing Id1 were utilized to regulate the levels of Id1 in SGC-7901 cells. RT-PCR and western blotting showed that the levels of Id1 were successfully regulated by Id1-siRNA and pcDNA3.1-Id1. In this study, the downregulation of Id1 in SGC-7901 cells inhibited cell proliferation and decreased the proportion of $\mathrm{G} 2 / \mathrm{M}$ phase of the cell cycle, while the upregulation of Id1 did not show these effects; the reason has yet to be determined. However, both the downregulation and upregulation of Id1 levels changed the levels of several cell cycle-related genes in this study, which is consistent with other recent reports (14,23-25). Our data suggest that Id1 is not involved in the apoptosis of SGC-7901 cells, but that the apoptosis-associated genes Bax and Bad are affected by Id1 levels. Therefore, the downregulation of Id1 did not elevate the levels of Bax and Bad to the extent that cell apoptosis occurred.

p-Akt is known to be involved in cell proliferation in several tumor models. We investigated whether $\mathrm{p}-\mathrm{Akt}$
(Thr308, Ser473) was involved in Id1-associated proliferation of SGC-7901 cells. Our findings regarding p-Akt in SGC-7901 cells are in agreement with those of other studies in that the downregulation of Id1 was capable of decreasing the levels of p-Akt (Thr308, Ser473).

In conclusion, our findings have shown for the first time that the Akt pathway is involved in Id1 in the proliferation of gastric cancer cells. Therefore, targeting Id1 may be a novel strategy for the treatment of gastric cancer.

\section{Acknowledgements}

This study was supported by the National Natural Science Foundation of China (Grant no. 30672358 to Zhenyu Zhu).

\section{References}

1. Jemal A, Bray F, Center MM, Ferlay J, Ward E and Forman D: Global Cancer Statistics. CA Cancer J Clin 61: 69-90, 2011.

2. Neugut AI, Hayek M and Howe G: Epidemiology of gastric cancer. Semin Oncol 23: 281-291, 1996.

3. Prinz C, Schwendy S and Voland P: H. pylori and gastric cancer: shifting the global burden. World J Gastroenterol 12: 54-58, 2006.

4. Roder DM: The epidemiology of gastric cancer. Gastric Cancer 5 (Suppl 1): 5-11, 2002.

5. Sikder HA, Devlin MK, Dunlap S, Ryu B and Alani RM: Id proteins in cell growth and tumorigenesis. Cancer Cell 3: 525-530, 2003.

6. Ruzinova MB and Benezra R: Id proteins in development, cell cycle and cancer. Trends Cell Biol 13: 410-418, 2003.

7. Perk J, Gil-Bazo I and Chin Y: Reassessment of Id1 protein expression in human mammary, prostate, and bladder cancers using a monospecific rabbit monoclonal anti-Id1 antibody. Cancer Res 66: 10870-10877, 2006.

8. Sun XH, Copeland NG, Jenkins NA and Baltimore D: Id proteins Id1 and Id2 selectively inhibit DNA binding by one class of helixloop-helix proteins. Mol Cell Biol 11: 5603-5611, 1991.

9. Lyden D, Young AZ and Zagzag D: Id1 and Id3 are required for neurogenesis, angiogenesis and vascularization of tumour xenografts. Nature 401: 670-677, 1999.

10. Desprez PY, Hara E, Bissell MJ and Campisi J: Suppression of mammary epithelial cell differentiation by the helix-loop-helix protein Id-1. Mol Cell Biol 15: 3398-3404, 1995.

11. Benezra R, Rafii S and Lyden D: The Id proteins and angiogenesis. Oncogene 20: 8334-8341, 2001.

12. Alani RM, Young AZ and Shifflett CB: Id1 regulation of cellular senescence through transcriptional repression of p16/Ink4a. Proc Natl Acad Sci USA 98: 7812-7816, 2001.

13. Ciarrocchi A, Jankovic V and Shaked Y: Id1 restrains p21 expression to control endothelial progenitor cell formation. PLoS ONE 2: e1338, 2007. 
14. Cheng YJ, Tsai JW, Hsieh KC, Yang YC, Chen YJ, Huang MS and Yuan SS: Id1 promotes lung cancer cell proliferation and tumor growth through Akt-related pathway. Cancer Lett 307: 191-199, 2011.

15. Parrinello S, Lin CQ and Murata K: Id-1, ITF-2, and Id-2 comprise a network of helix-loop-helix proteins that regulate mammary epithelial cell proliferation, differentiation, and apoptosis. J Bio Chem 276: 39213-39219, 2001.

16. Tanaka K, Pracyk JB and Takeda K: Expression of Id1 results in apoptosis of cardiac myocytes through a redox-dependent mechanism. J Biol Chem 273: 25922-25928, 1998.

17. Yang HY, Liu HL and Jiang HC: Expression and prognostic values of Id-1 and Id-3 in gastric adenocarcinoma. J Surg Res 167: 258-266, 2011

18. Wang Q, Tsao SW and Wang X: Overexpression of Id-1 in gastric adenocarcinoma: implication for a novel diagnostic marker. Anticancer Res 24: 881-886, 2004.

19. Tsuchiya T, Okaji Y, Tsuno NH and Sakurai D: Targeting Id1 and Id3 inhibits peritoneal metastasis of gastric cancer. Cancer Sci 96: 784-790, 2005.

20. Zhang Y, Han J, Yang X, Shao C, Xu Z, Cheng R, Cai W, Ma J, Yang Z and Gao G: Pigment epithelium-derived factor inhibits angiogenesis and growth of gastric carcinoma by downregulation of VEGF. Oncol Rep 26: 681-686, 2011.
21. Ling YX, Tao J, Fangc SF, Hui Z and Fang QR: Downregulation of Id1 by small interfering RNA in prostate cancer PC 3 cells in vivo and in vitro. Eur J Cancer Prev 20: 9-17, 2011.

22. Gautschi O, Tepper CG and Purnell PR: Regulation of Id1 expression by Src: implications for targeting of the bone morphogenetic protein pathway in cancer. Cancer Res 68: 2250-2258, 2008.

23. Geng H, Rademacher BL and Pittsenbarger J: ID1 enhances docetaxel cytotoxicity in prostate cancer cells. Cancer Res 70: 3240-3248, 2010.

24. Li B, Tsao SW, Li YY, Wang X and Cheung ALM: Id-1 promotes tumorigenicity and metastasis of human esophageal cancer cells through activation of PI3K/AKT signaling pathway. Int J Cancer 125: 2576-2585, 2009.

25. Swarbrick A, Akerfeldt MC, Lee CSL and Musgrove EA Regulation of cyclin expression and cell cycle progression in breast epithelial cells by the helix-loop-helix protein Id1. Oncogene 24: 381-389, 2005. 